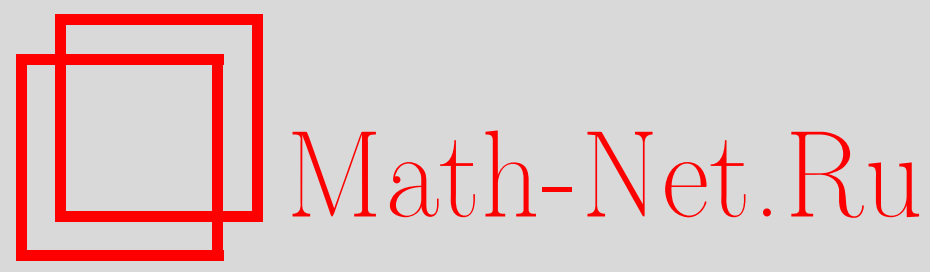

И. К. Шаранхаев, О бесповторных булевых функциях в предэлементарных монотонных базисах, Дискрет. матем., 2009, том 21, выпуск 2, 88-93

DOI: https://doi.org/10.4213/dm1048

Использование Общероссийского математического портала Math-Net.Ru подразумевает, что вы прочитали и согласны с пользовательским соглашением http://www.mathnet.ru/rus/agreement

Параметры загрузки:

IP: 34.239 .49 .27

26 апреля 2023 г., 16:18:02 


\title{
О бесповторных булевых функциях в предэлементарных монотонных базисах
}

\author{
() 2009 г. И. К. Шаранхаев
}

\begin{abstract}
Изучается представление булевых функций формулами. Описаны в терминах остаточных функций булевы функции, реализуемые бесповторными формулами в предэлементарных базисах $\left\{\vee, \cdot,-, 0,1, x_{1}\left(x_{2} \vee \ldots \vee x_{n}\right) \vee x_{2} \cdot \ldots \cdot x_{n}\right\}$, где $n \geqslant 4$.

Работа выполнена при поддержке грантом Бурятского государственного университета.
\end{abstract}

\section{1. Введение}

Под базисами понимаем конечные полные множества булевых функций, содержащие константы.

Формула $\Phi$ над базисом $B$ называется бесповторной, если каждая переменная входит в нее не более одного раза. Булева функция $f$ называется бесповторной в базисе $B$, если существует бесповторная формула $\Phi$ над $B$, представляющая функцию $f$. В противном случае $f$ называют повторной в $B$.

Настоящая работа посвящена нахождению условий, равносильных бесповторности булевых функций в базисах $\left\{\vee, \cdot,-, 0,1, x_{1}\left(x_{2} \vee \ldots \vee x_{n}\right) \vee x_{2} \cdot \ldots \cdot x_{n}\right\}$, где $n \geqslant 4$. Дадим необходимые определения и обозначения, все неопределяемые здесь понятия можно найти, например, в [1]. Будут использоваться следующие обозначения: переменные обозначаются символами $x, y, z, u, v$, возможно с индексами; $\sigma, \sigma_{1}, \ldots, \sigma_{n}$ - булевы константы; символом $\tilde{x}$ обозначается набор $\left(x_{1}, \ldots, x_{n}\right) ;|\tilde{x}|$ - длина набора $\tilde{x} ; P_{B}-$ множество всех бесповторных функций в базисе $B$;

$$
x^{\sigma}= \begin{cases}x, & \text { если } \sigma=1, \\ \bar{x}, & \text { если } \sigma=0\end{cases}
$$

Функция, получаемая из $f\left(x_{1}, \ldots, x_{n}\right)$ подстановкой вместо некоторой переменной $x_{i}$ константы $\sigma$, называется остаточной и обозначается $f_{x_{i}}^{\sigma}$. Индуктивно это определение распространяется на подмножество переменных.

Назовем переменную $x_{i}$ функции $f$ фиктивной, если $f_{x_{i}}^{0}=f_{x_{i}}^{1}$, и существенной в противном случае. Множество всех существенных переменных функции $f$ обозначим через $\rho(f)$, а множество всех фиктивных переменных функции $f$ - через $\delta(f)$.

Рангом rank $f$ функции $f$ называется число ее существенных переменных. Под рангом базиса понимаем наибольший из рангов, входящих в него функций. 
Функцией $f$ называется слабоповторной в базисе $B$, если любая остаточная функция от функции $f$ является бесповторной, а сама $f$ повторна в базисе $B$. Через $S_{B}$ обозначим множество всех слабоповторных функций в базисе $B$. Базис $B_{0}=\{\vee, \cdot,-, 0,1\}$ называется элементарным, а базис $B_{0} \cup\{f\}$, где $f$ слабоповторна в $B_{0}$, называется предэлементарным.

В работе [2] на множестве всех базисов вводится частичный порядок следующим образом: базис $B$-меньше базиса $C$ (в этом случае пишем $B \leqslant_{p} C$ ), если $P_{B} \subseteq P_{C}$. Если $B \leqslant{ }_{p} C$ и $C \leqslant \leqslant_{p} B$, то базисы $B$ и $C$ называются $p$-эквивалентными $\left(B \sim_{p} C\right)$. Если $B \leqslant_{p} C$ и $B \nsim_{p} C$, то базис $B$ строго $p$-меньше базиса $C$ (пишем $B<_{p} C$ ). Базис $B$ непосредственно $p$-меньше базиса $C$ (пишем $B \triangleleft_{p} C$ ), если $B<_{p} C$ и не существует базиса $D$ такого, что $B<_{p} D<_{p} C$. Там же доказано, что для любого базиса $B$ верно соотношение $B_{0} \leqslant_{p} B$, а соотношение $C \triangleleft_{p} D$ выполняется тогда и только тогда, когда $D \sim_{p} C \cup\{f\}$, где $f \in S_{C}$.

Отметим, что частично упорядоченное множество базисов с введенным порядком дуально изоморфно частично упорядоченному множеству базисов, рассматриваемому в [3], где базисы сравниваются по сложности формульных представлений.

Описание всех предэлементарных базисов (с точностью до эквивалентности $\sim_{p}$ ) следует из [4]. Введем обозначения для таких базисов:

$$
\begin{aligned}
& B_{1, n}=B_{0} \cup\left\{g_{1, n}\right\}, \quad g_{1, n}=x_{1} \cdot \ldots \cdot x_{n} \vee \bar{x}_{1} \cdot \ldots \cdot \bar{x}_{n}, \quad n \geqslant 2 ; \\
& B_{2, n}=B_{0} \cup\left\{g_{2, n}\right\}, \quad g_{2, n}=x_{1}\left(x_{2} \vee \ldots \vee x_{n}\right) \vee x_{2} \cdot \ldots \cdot x_{n}, \quad n \geqslant 3 \text {; } \\
& B_{3, n}=B_{0} \cup\left\{g_{3, n}\right\}, \quad g_{3, n}=x_{1}\left(x_{2} \vee x_{3} \cdot \ldots \cdot x_{n}\right) \vee x_{2} \bar{x}_{3} \cdot \ldots \cdot \bar{x}_{n}, \quad n \geqslant 3 \text {; } \\
& B_{4}=B_{0} \cup\left\{g_{4}\right\}, \quad g_{4}=x_{1}\left(x_{2} \vee x_{3}\right) \vee x_{3} x_{4} ; \\
& B_{5}=B_{0} \cup\left\{g_{5}\right\}, \quad g_{5}=x_{1}\left(x_{2} \vee x_{3} x_{4}\right) \vee x_{5}\left(x_{3} \vee x_{2} x_{4}\right) \text {. }
\end{aligned}
$$

Впервые критерий бесповторности булевых функций был получен Б. А. Субботовской для элементарного базиса $B_{0}$. В [2, 5-10] можно найти все известные критерии бесповторности булевых функций, найденные для базисов $B_{0}, B_{2,3}, B_{3,3}$ и $B_{1, n}$, где $n$ - нечетное число, большее 1 . В данной статье результат для базиса $B_{2,3}$ (см. теорему 4 в [2]) обобщен для всей последовательности базисов $B_{2, n}$.

Базис $B_{2, n}$ будем называть предэлементарным монотонным базисом ранга $n$.

Будем говорить, что функции $f$ и $g$ связаны отношением $\preccurlyeq$, и писать $f \preccurlyeq g$, если для любого набора $\tilde{\sigma}$ выполняется неравенство $f(\tilde{\sigma}) \leqslant g(\tilde{\sigma})$.

Функция $f$ называется обобщенно монотонной по переменной $x$, если выполняется либо $f_{x}^{0} \preccurlyeq f_{x}^{1}$, либо $f_{x}^{0} \succcurlyeq f_{x}^{1}$. Если функция $f$ является обобщенно монотонной по переменной $x$, то для краткости будем писать $f \in M_{x}$.

Функция $f$ называется монотонной, если для любой переменной $x$ выполняется соотношение $f_{x}^{0} \preccurlyeq f_{x}^{1}$.

Функции $f$ и $g$ называются обобщенно однотипными, если выполняется соотношение $f\left(x_{1}, \ldots, x_{n}\right)=g^{\sigma}\left(x_{i_{1}}^{\sigma_{1}}, \ldots, x_{i_{n}}^{\sigma_{n}}\right)$, где $\left(i_{1}, \ldots, i_{n}\right)-$ некоторая перестановка чисел от 1 до $n$. Очевидно, что на множестве всех булевых функций отношение обобщенной однотипности является отношением эквивалентности.

Производной функции $f\left(x_{1}, \ldots, x_{n}\right)$ по переменной $x_{i}$ называется функция $f_{x_{i}}^{\prime}=f_{x_{i}}^{0} \oplus f_{x_{i}}^{1}$.

Понятие производной функции по переменной распространяется индуктивно на множество переменных следующим образом:

$$
\frac{\partial f}{\partial x_{i_{1}} \ldots \partial x_{i_{s}}}=\frac{\partial\left(\partial f /\left(\partial x_{i_{1}} \ldots \partial x_{i_{s-1}}\right)\right)}{\partial x_{i_{s}}} .
$$


Функция называется нечетной, если число наборов, на которых функция равна 1, является нечетным, и четной в противном случае.

Множество булевых функций $P$, содержащее тождественную функцию, называется наследственным, если для любой функции $f \in P$ любая остаточная функция $f_{x}^{\sigma} \in P$.

Множество булевых функций $P$ называется инвариантным, если для любых функций $f(\tilde{u}, y), g(\tilde{v}) \in P$, где $\tilde{u} \cap \tilde{v}=\varnothing$, справедливо включение $f(\tilde{u}, g(\tilde{v})) \in P$.

\section{2. Вспомогательные утверждения}

При доказательстве основного результата будут использоваться следующие утверждения.

Предложение 1 ([9]). Множество булевых функиий $P$ является наследственным и инвариантным тогда и только тогда, когда $P$ есть множество всех бесповторных функиий над некоторым базисом В.

Следствие 1. Если для наследственного, инвариантного множества булевых функиий $P$ и базиса $B$ верно, что $B \subseteq P$ и $S_{B} \cap P=\varnothing$, то $P_{B}=P$.

Таким образом, для доказательства того, что некоторое множество булевых функций $P$ совпадает с множеством всех бесповторных функций над некоторым базисом $B$, достаточно показать, что $P$ обладает свойствами наследственности и инвариантности, и проверить, что все слабоповторные в $B$ функции не входят в $P$.

Предложение 2 ([11]). Следующая система булевых функицй является полной системой представителей классов эквивалентности по отношению обобщенной однотипности для булевых функиий, слабоповторных в предэлементарном базисе $B_{2, n}$, где $n \geqslant 4$ :

$$
\begin{array}{rlrl}
x_{1}\left(x_{2} \vee x_{3}\right) & \vee x_{3} x_{4}, & & \\
x_{1}\left(x_{2} \vee x_{3} x_{4}\right) & \vee x_{5}\left(x_{3} \vee x_{2} x_{4}\right), & & \\
x_{1}\left(x_{2} \vee \ldots \vee x_{k}\right) & \vee x_{2} \cdot \ldots \cdot x_{k}, & k \geqslant 3, \quad k \neq n, \\
x_{1}\left(x_{2} \vee x_{3} \cdot \ldots \cdot x_{k}\right) & \vee x_{2} \bar{x}_{3} \cdot \ldots \cdot \bar{x}_{k}, & k \geqslant 3, & \\
x_{1} \cdot \ldots \cdot x_{k} & \vee \bar{x}_{1} \cdot \ldots \cdot \bar{x}_{k}, & & \\
\bar{x}_{1} g\left(x_{2}, \ldots, x_{n+1}\right) & \vee x_{1} x_{2}\left(x_{3} \vee \ldots x_{n+1}\right), & & \\
\bar{x}_{1} g\left(x_{2}, \ldots x_{n+1}\right) & \vee x_{1} \cdot \ldots \cdot x_{n+1}, & & \\
\bar{x}_{1} g\left(x_{2}, \ldots, x_{n+1}\right) & \vee x_{1} x_{2} . &
\end{array}
$$

\section{3. Основной результат}

В этом разделе будут получены критерии бесповторности булевых функций для предэлементарных монотонных базисов.

Функцию $f$ будем называть $n$-нежесткой, где $n \geqslant 2$, если либо $\operatorname{rank} f<2$, либо для любого $x \in \rho(f)$ справедливо включение $f \in M_{x}$ и выполняется одно из условий:

(1) $\delta(f)=\delta\left(f_{x}^{0}\right)$ и $\delta(f) \subsetneq \delta\left(f_{x}^{1}\right)$;

(2) $\delta(f)=\delta\left(f_{x}^{1}\right)$ и $\delta(f) \subsetneq \delta\left(f_{x}^{0}\right)$; 
(3) существуют $y_{1}, \ldots, y_{n-1} \in \rho\left(f_{x}^{\prime}\right)$ такие, что справедливо строгое включение

$$
\delta\left(f_{x}^{\prime}\right) \subsetneq \delta\left(\frac{\partial f_{x}^{\prime}}{\partial y_{1} \ldots \partial y_{n-1}}\right)
$$

и при $n=4$ для любых $i$ и $j$ справедливо соотношение $\partial f_{x}^{\prime} / \partial y_{i} \notin M_{y_{j}}$, где $1 \leqslant i, j \leqslant 3$ и $i \neq j$.

Функцию $f$ будем называть наследственно $n$-нежесткой, если сама $f$ и все ее остаточные функции являются $n$-нежесткими.

Теорема 1. Функция $f$ бесповторна в базисе $B_{2, n}$ тогда и только тогда, когда она является наследственно $(n-1)$-нежесткой.

Доказательство. Для доказательства теоремы воспользуемся методом, основанным на предложении 1. Так как случай $n=3$ рассмотрен в [2], будем считать, что $n \geqslant 4$.

Обозначим через $P_{n-1}$ множество всех наследственно $(n-1)$-нежестких функций. Множество $P_{n-1}$ является наследственным по определению, покажем его инвариантность.

Пусть $f(\tilde{u}, \tilde{v})=g(\tilde{u}, h(\tilde{v}))$, где $g(\tilde{u}, y), h(\tilde{v}) \in P_{n-1}$. Если $\tilde{u}=\varnothing$ или $|\tilde{v}|=1$, то функция $f$ обобщенно однотипна с функцией $g$ или $h$, поэтому является наследственно $(n-1)$-нежесткой. Далее считаем, что $\tilde{u} \neq \varnothing$ и $|\tilde{v}|>1$.

1. Пусть $x \in \tilde{v}$. Если выполняется одно из строгих включений $\delta(h) \subsetneq \delta\left(h_{x}^{0}\right)$ или $\delta(h) \subsetneq \delta\left(h_{x}^{1}\right)$, то соответственно либо $\delta(f) \subsetneq \delta\left(f_{x}^{0}\right)$, либо $\delta(f) \subsetneq \delta\left(f_{x}^{1}\right)$. В противном случае найдутся переменные $x_{1}, \ldots, x_{n-2} \in \rho\left(h_{x}^{\prime}\right)$ такие, что

$$
\delta\left(h_{x}^{\prime}\right) \subsetneq \delta\left(\frac{\partial h_{x}^{\prime}}{\partial x_{1} \ldots \partial x_{n-2}}\right) .
$$

Так как $f_{x}^{\prime}=g_{y}^{\prime}(\tilde{u}, y) h_{x}^{\prime}(\tilde{v})$ и

$$
\frac{\partial f_{x}^{\prime}}{\partial x_{1} \ldots \partial x_{n-2}}=g_{y}^{\prime}(\tilde{u}, y) \frac{\partial h_{x}^{\prime}}{\partial x_{1} \ldots \partial x_{n-2}},
$$

справедливо строгое включение

$$
\delta\left(f_{x}^{\prime}\right) \subsetneq \delta\left(\frac{\partial f_{x}^{\prime}}{\partial x_{1} \ldots \partial x_{n-2}}\right) .
$$

2. Пусть $x \in \tilde{u}$. Если справедливо одно из строгих включений $\delta(g) \subsetneq \delta\left(g_{x}^{0}\right)$ или $\delta(g) \subsetneq \delta\left(g_{x}^{1}\right)$, то справедливость ровно одного из строгих включений $\delta(f) \subsetneq \delta\left(f_{x}^{0}\right)$ или $\delta(f) \subsetneq \delta\left(f_{x}^{1}\right)$ очевидна. В противном случае рассмотрим $f_{x}^{\prime}=g_{x}^{\prime}(\tilde{u}, h(\tilde{v}))$. Если для функции $g_{x}^{\prime}(\tilde{u}, y)$ существуют переменные $x_{1}, \ldots, x_{n-2} \in \rho\left(g_{x}^{\prime}(\tilde{u}, y)\right)$, отличные от $y$ такие, что

$$
\delta\left(g_{x}^{\prime}\right) \subsetneq \delta\left(\frac{\partial g_{x}^{\prime}}{d x_{1} \ldots \partial x_{n-2}}\right),
$$

то справедливо строгое включение

$$
\delta\left(f_{x}^{\prime}\right) \subsetneq \delta\left(\frac{\partial f_{x}^{\prime}}{d x_{1} \ldots \partial x_{n-2}}\right) .
$$

В противном случае существуют $y_{1}, \ldots, y_{n-3} \in \rho\left(g_{x}^{\prime}(\tilde{u}, y)\right)$, отличные от $y$ такие, что справедливо строгое включение

$$
\delta\left(g_{x}^{\prime}\right) \subsetneq \delta\left(\frac{\partial g_{x}^{\prime}}{d y_{1} \ldots \partial y_{n-3} d y}\right) .
$$


Далее выберем произвольным образом существенную переменную $z \in \tilde{v}$ и рассмотрим равенство

$$
\frac{\partial f_{x}^{\prime}}{\partial y_{1} \ldots \partial y_{n-3} \partial z}=\frac{\partial g_{x}^{\prime}}{\partial y_{1} \ldots \partial y_{n-3} \partial y} h_{z}^{\prime}(\tilde{v}) .
$$

В силу того, что

$$
\delta\left(g_{x}^{\prime}\right) \subsetneq \delta\left(\frac{\partial g_{x}^{\prime}}{\partial y_{1} \ldots \partial y_{n-3} \partial y}\right),
$$

справедливо строгое включение

$$
\delta\left(f_{x}^{\prime}\right) \subsetneq \delta\left(\frac{\partial f_{x}^{\prime}}{d y_{1} \ldots \partial y_{n-3} \partial z}\right) .
$$

Таким образом, доказано, что если для некоторой переменной $x \in \rho\left(f_{x}^{\prime}\right)$ не выполняются условия

(1) $\delta(f)=\delta\left(f_{x}^{0}\right)$ и $\delta(f) \subsetneq \delta\left(f_{x}^{1}\right)$,

(2) $\delta(f)=\delta\left(f_{x}^{1}\right)$ и $\delta(f) \subsetneq \delta\left(f_{x}^{0}\right)$,

тогда существуют переменные $z_{1}, \ldots, z_{n-2} \in \rho\left(f_{x}^{\prime}\right)$ такие, что

$$
\delta\left(f_{x}^{\prime}\right) \subsetneq \delta\left(\frac{\partial f_{x}^{\prime}}{\partial z_{1} \ldots \partial z_{n-3}}\right) .
$$

По ходу доказательства нетрудно заметить, что $\partial f_{x}^{\prime} / \partial z_{i} \notin M_{z_{j}}$ при $n=5$ для любых $i$ и $j$, где $1 \leqslant i, j \leqslant 3$ и $i \neq j$.

Доказательство того, что $f \in M_{x}$ для любого $x$ проводится аналогично случаю $n=3$, рассмотренному в [2]. Таким образом, доказана инвариантность $P_{n-1}$.

Теперь для наследственного инвариантного множества $P_{n-1}$ найдется порождающий его базис. Очевидно, что $B_{2, n} \subseteq P_{n-1}$. Проверим, что все слабоповторные функции в базисе $B_{2, n}$ не принадлежат $P_{n-1}$. Достаточно ограничиться проверкой функций из предложения 2, так как если свойство $(n-1)$-нежесткости не выполняется для некоторой функции, то оно не выполняется и для всех обобщенно однотипных с ней функций.

(a) Пусть $f=x_{1}\left(x_{2} \vee x_{3}\right) \vee x_{3} x_{4}$. Тогда $f_{x_{2}}^{0}=x_{3}\left(x_{1} \vee x_{4}\right), f_{x_{2}}^{1}=x_{1} \vee x_{3} x_{4}$, $f_{x_{2}}^{\prime}=x_{1} \bar{x}_{3}$.

Множество $\rho\left(f_{x_{2}}^{\prime}\right)=\left\{x_{1}, x_{3}\right\}$ и $\delta\left(f_{x_{2}}^{\prime}\right)=\delta\left(\partial f_{x_{2}}^{\prime} / \partial x_{1}\right), \delta\left(f_{x_{2}}^{\prime}\right)=\delta\left(\partial f_{x_{2}}^{\prime} / \partial x_{3}\right)$, $\delta\left(f_{x_{2}}^{\prime}\right)=\delta\left(\partial f_{x_{2}}^{\prime} / \partial x_{1} \partial x_{3}\right)$, поэтому $f \notin P_{n-1}$.

(б) Пусть $f=x_{1}\left(x_{2} \vee x_{3} x_{4}\right) \vee x_{5}\left(x_{3} \vee x_{2} x_{4}\right)$. Тогда $f_{x_{1}}^{0}=x_{5}\left(x_{3} \vee x_{2} x_{4}\right)$, $f_{x_{1}}^{1}=x_{2} \vee x_{3}\left(x_{4} \vee x_{5}\right), f_{x_{1}}^{\prime}=x_{2}\left(\bar{x}_{5} \vee \bar{x}_{3} \bar{x}_{4}\right) \vee \bar{x}_{5} x_{3} x_{4}$

Легко видеть, что справедливы включения $f_{x_{1}}^{\prime} \in M_{x_{2}}$ и $f_{x_{1}}^{\prime} \in M_{x_{5}}$. Прямая проверка показывает, что существуют ровно три переменные $z_{1}, z_{2}, z_{3} \in \rho\left(f_{x_{1}}^{\prime}\right)$ такие, что $\delta\left(f_{x_{1}}^{\prime}\right) \subsetneq \delta\left(\partial f_{x_{1}}^{\prime} /\left(\partial z_{1} \partial z_{2} \partial z_{3}\right)\right)$, то есть одной из переменных является либо $x_{2}$, либо $x_{5}$. Но $f_{x_{1}}^{\prime} \in M_{x_{2}}$ и $f_{x_{1}}^{\prime} \in M_{x_{5}}$, поэтому $f \notin P_{n-1}$.

(в) Пусть $f=x_{1} \ldots x_{k} \vee \bar{x}_{1} \ldots \bar{x}_{k}$, где $k \geqslant 2$. Функция $f \notin P_{n-1}$, так как $f \notin M_{x_{1}}$.

(г) Пусть $f=x_{1}\left(x_{2} \vee x_{3} \ldots x_{k}\right) \vee x_{2} \bar{x}_{3} \ldots \bar{x}_{k}$, где $k \geqslant 3$. Ситуация аналогична (в).

(д) Пусть $f=x_{1}\left(x_{2} \vee \ldots x_{k}\right) \vee x_{2} \ldots x_{k}$, где $k \geqslant 3$ и $k \neq n$. Тогда $f_{x_{1}}^{0}=x_{2} \ldots x_{k}$, $f_{x_{1}}^{1}=x_{2} \vee \ldots \vee x_{k}, f_{x_{1}}^{\prime}=\overline{x_{2} \ldots x_{k} \vee \bar{x}_{2} \ldots \bar{x}_{k}}$.

Легко заметить, что существуют ровно $k-2$ переменные $z_{1}, \ldots, z_{k-2}$ такие, что $\delta\left(f_{x_{1}}^{\prime}\right) \subsetneq \delta\left(\partial f_{x_{1}}^{\prime} / \partial z_{1} \ldots \partial z_{k-2}\right)$. Так как $k \neq n$, функция $f \notin P_{n-1}$. 
(е) Пусть $f=\bar{x}_{1} g\left(x_{2}, \ldots, x_{n+1}\right) \vee x_{1} x_{2}$. Так как $f_{x_{1}}^{0} \not f_{x_{1}}^{1}$ и $f_{x_{1}}^{0} \nsucceq_{x_{1}}^{1}$, то $f \notin M_{x_{1}}$, поэтому $f \notin P_{n-1}$.

(ж) Пусть $f=\bar{x}_{1} g\left(x_{2}, \ldots, x_{n+1}\right) \vee x_{1} \ldots x_{n+1}$. Тогда $f_{x_{1}}^{0}=g\left(x_{2}, \ldots, x_{n+1}\right)$, $f_{x_{1}}^{1}=x_{2} \ldots x_{n+1}, f_{x_{1}}^{\prime}=g\left(x_{2}, \ldots, x_{n+1}\right) \oplus x_{2} \ldots x_{n+1}$.

Функция $g\left(x_{2}, \ldots, x_{n+1}\right)$ является четной, причем в ее совершенной дизъюнктивной нормальной форме есть конъюнкция $x_{2} \ldots x_{n+1}$, поэтому функция $f_{x_{1}}^{\prime}$ нечетна, то есть существенна. Очевидно, что производная нечетной функции по любой переменной есть нечетная функция, поэтому $f \notin P_{n-1}$.

(3) Пусть $f=\bar{x}_{1} g\left(x_{2}, \ldots, x_{n+1}\right) \vee x_{1} x_{2}\left(x_{3} \vee \ldots \vee x_{n+1}\right)$. Тогда $f_{x_{1}}^{0}=g\left(x_{2}, \ldots, x_{n+1}\right)$, $f_{x_{1}}^{1}=x_{2}\left(x_{3} \vee \ldots \vee x_{n+1}\right), f_{x_{1}}^{\prime}=g\left(x_{2}, \ldots, x_{n+1}\right) \oplus x_{2}\left(x_{3} \vee \ldots x_{n+1}\right)=\bar{x}_{2} x_{3} \ldots x_{n+1}$.

Функция $f_{x_{1}}^{\prime}$ является нечетной. Ситуация аналогична случаю (е).

Таким образом, $S_{B_{2, n}} \cap P_{n-1}=\varnothing, B_{2, n} \subseteq P_{n-1}$. Теорема доказана.

\section{Список литературы}

1. Винокуров С. Ф., Перязев Н. А., Избранные вопросы теории булевых функций. Физматлит, Москва, 2001.

2. Перязев Н. А., Шаранхаев И. К., Критерии бесповторности булевых функций в предэлементарных базисах ранга 3. Дискретная математика (2005) 17, №2, 127-138.

3. Черухин Д. Ю., Алгоритмический критерий сравнения булевых базисов. Матем. вопр. киберн. (1999) 8, 77-122.

4. Стеценко В. А., О предплохих базисах в $P_{2}$. Матем. вопр. киберн. (1992) 4, 139-177.

5. Субботовская Б. А.,, О сравнении базисов при реализации функций алгебры логики формулами. Докл. АН СССР (1963) 149, №4, 784-787.

6. Гуревич В. А., Критерии бесповторности функций алгебры логики. Докл. АН СССР (1991) $\mathbf{3 1 8}$, №3, 532-537.

7. Перязев Н. А., Реализация булевых функций бесповторными формулами в некоторых базисах. В сб.: Алгебра, логика и приложения, Иркутск, 1994, с. 134-154.

8. Перязев Н. А., Реализация булевых функций бесповторными формулами. Дискретная математика (1995) 7, №3, 61-68.

9. Кириченко К. Д., О критериях бесповторности булевых функций в различных базисах. Onтuмизачия, управление, интеллект (2000) 4, 83-101.

10. Шаранхаев И. К., О представлении булевых функций бесповторными формулами в некоторых симметрических базисах. Вестник Бурятского гос. унив. Сер. матем., информ., физ., техн. (2007) 6, 53-56.

11. Кириченко К. Д., Слабоповторные булевы функции в некоторых предэлементных базисах. В сб.: Дискретная математика и информатика, 13. Изд-во Иркутского унив., Иркутск, 2000.

Статья поступила 19.09.2006. Переработанный вариант поступил 15.10.2007. 